Leser der MMW können sich mit allen Fragen zur Abrechnung und Praxisführung an Helmut Walbert, Facharzt für Allgemeinmedizin, Würzburg, wenden. Sie erreichen inn jeden Donnerstag von 13 bis 15 Uhr unter der kostenlosen Rufnummer (0800) 2379830 oder per E-Mail: w@lbert.info.
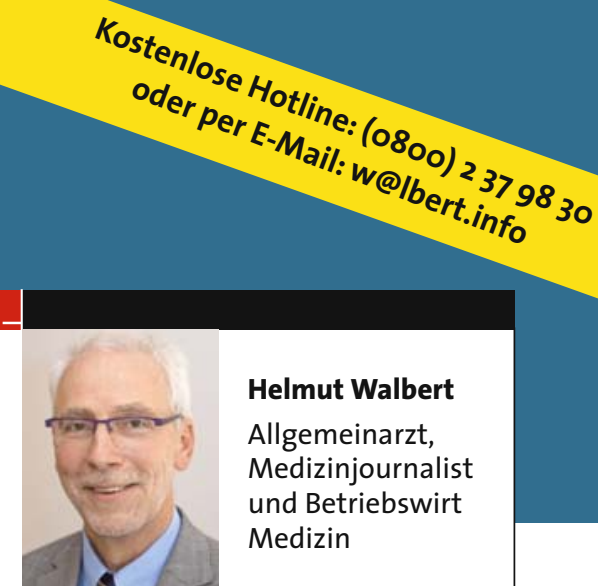

Helmut Walbert

Allgemeinarzt, Medizinjournalist und Betriebswirt Medizin

\title{
Geht das auch telefonisch?
}

Frage von Dr. H., Allgemeinarzt, KV Bayern Kann ich die Gebührenordnungsposition 4, Erhebung der Fremdanamnese über einen Kranken, auch telefonisch erbringen und abrechnen?

Antwort: Grundsätzlich ja. Die GOP 4 ist mit 220 Punkten, entsprechend $12,82 €$ GOÄ einfach, jedoch deutlich höher bewertet als die GOP 1 mit 80 Punkten.

Aus diesem Grund ist der Leistungsinhalt der GOP 4 auch deutlich aufwendiger: „Erhebung der Fremdanamnese über ei- nen Kranken und/oder Unterweisung und Führung der Bezugsperson im Zusammenhang mit der Behandlung eines Kranken“ Dies bedeutet, dass die kurze Befragung zum Krankheitsverlauf, zur Symptomatik der Erkrankung und die entsprechende Therapieanweisung, insbesondere wenn es sich um ein- und dieselbe Bezugsperson handelt, hier nicht gemeint sind. Dies wäre der normale Leistungsinhalt der GOP 1. Dies hat bereits das LG Karlsruhe unter dem Aktenzeichen 1 S 90/99 vom 14.3.2001 festgestellt.
Die GOP 4 vergütet die aufwendigere Fremdanamnese im Sinne einer grundsätzlichen, umfangreicheren Befragung und/oder Unterweisung und Führung der Bezugsperson. Dies ist auch der Grund, warum die GOP 4 nur einmal im Behandlungsfall abgerechnet werden kann. Lediglich bei zwei Erkrankungen innerhalb des Monats kann auch ein zweimaliger Ansatz infrage kommen.

\section{Altenheimvisite bei einem dementen Patienten mit Hypertonie, Diabetes und Apoplex}

\section{Gibt es hier einen Zuschlag?}

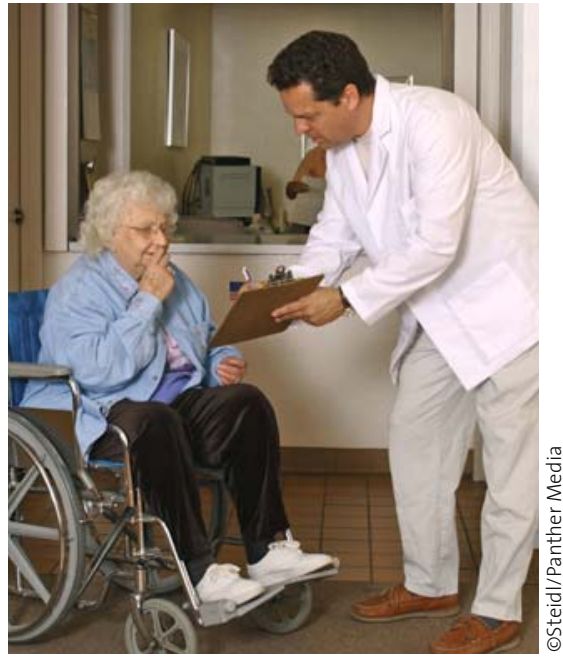

Persönlicher Arzt-Patienten-Kontakt ist auch für die Abrechnung entscheidend.
Frage von Dr. med. H.S. Allgemeinarzt, Durlangen:

Wenn ich im Altenheim Visite bei einem Patienten mit Demenz, Hypertonie, Diabetes und Apoplex mache, kann ich hier den Zuschlag für die Behandlung eines Versicherten mit einer oder mehreren schwerwiegenden chronischen Erkrankung(en) abrechnen?

Antwort: Ja, selbstverständlich. Aber erst beim zweiten Arzt-Patienten-Kontakt (APK), da die GOP 03212 als obligaten Bestandteil zwei APKs hat.

Davon muss allerdings nur ein Kontakt ein sogenannter „persönlicher“ APK sein. Unter einem persönlichen APK wird die gleichzeitige Anwesenheit von Arzt und
Patient am gleichen Ort verstanden. Der zweite Kontakt kann auch ein telefonischer oder mittelbarer - über eine Bezugsperson - sein. Dieser kann vor Ihrer Visite oder nachher sein. Da diese Kontakte gebührenordnungsmäßig nicht mehr mit eigener Gebührenordnungsposition (GOP) erfasst werden, muss eine entsprechende Dokumentation in der Kartei des Patienten erfolgen. Die Visite, also den Besuch zum vorher vereinbarten Zeitpunkt mit Unterstützung des Pflegepersonals, rechnen Sie entweder als Einzelbesuch nach GOP 01 410 oder als Mitbesuch nach GOP 01413 $a b$. 